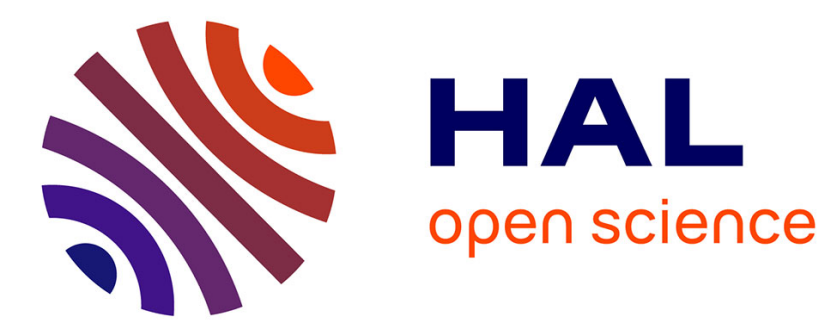

\title{
Preliminary study on launch vehicle design: Applications of multidisciplinary design optimization methodologies
}

\author{
Loïc Brevault, Mathieu Balesdent, Sebastien Defoort
}

\section{To cite this version:}

Loïc Brevault, Mathieu Balesdent, Sebastien Defoort. Preliminary study on launch vehicle design: Applications of multidisciplinary design optimization methodologies. Concurrent Engineering: Research and Applications, 2017, 26 (1), pp.1-11. 10.1177/1063293X17737131 . hal-01857722

\section{HAL Id: hal-01857722 \\ https://hal.science/hal-01857722}

Submitted on 17 Aug 2018

HAL is a multi-disciplinary open access archive for the deposit and dissemination of scientific research documents, whether they are published or not. The documents may come from teaching and research institutions in France or abroad, or from public or private research centers.
L'archive ouverte pluridisciplinaire HAL, est destinée au dépôt et à la diffusion de documents scientifiques de niveau recherche, publiés ou non, émanant des établissements d'enseignement et de recherche français ou étrangers, des laboratoires publics ou privés. 


\title{
Preliminary study on launch vehicle design - applications of Multidisciplinary Design Optimization methodologies
}

\author{
Loïc Brevault, Mathieu Balesdent, Sebastien Defoort \\ ONERA - The French Aerospace Lab \\ Chemin de la Hunière - 91123 Palaiseau, France \\ Email: firstname.lastname@onera.fr
}

\begin{abstract}
The design of complex systems such as launch vehicles involves different fields of expertise that are interconnected. To perform multidisciplinary studies, concurrent engineering aims at providing a collaborative environment which often relies on data set exchange. In order to efficiently achieve system level analyses (uncertainty propagation, sensitivity analysis, optimization, etc.) it is necessary to go beyond data set exchange which limits the capabilities of performance assessments. Multidisciplinary Design Optimization (MDO) methodologies is a collection of engineering methodologies to optimize systems modeled as a set of coupled disciplinary analyses and is a key enabler to extend concurrent engineering capabilities. This paper is focused on several examples of recent developments of MDO methodologies (e.g. MDO with transversal decomposition of the design process, MDO under uncertainty) with applications to launch vehicle design to illustrate the benefices of taking into account the coupling effects between the different physics all along the design process. These methods enable to manage the complexity of the involved physical phenomena and their interactions in order to generate innovative concepts such as reusable launch vehicles beyond existing solutions.
\end{abstract}

\section{INTRODUCTION}

The design of complex systems such as launch vehicles, satellites, aircraft, etc. involves different fields (disciplines) of expertise (e.g. aerodynamics, structure, trajectory) that interact. To design new aerospace vehicles, one of the challenges is to manage the complexity of the involved physical phenomena and their interactions in order to generate innovative concepts beyond existing solutions, with a generic methodological approach to be applied to very different systems such as launchers or aircraft. Concurrent engineering aims at providing a collaborative and simultaneous engineering working environment in order to perform multidisciplinary studies to design complex systems. The concurrent engineering approach relies on a process, a multidisciplinary team, an integrated design model, a facility and a software infrastructure (Xu et al., 2013).
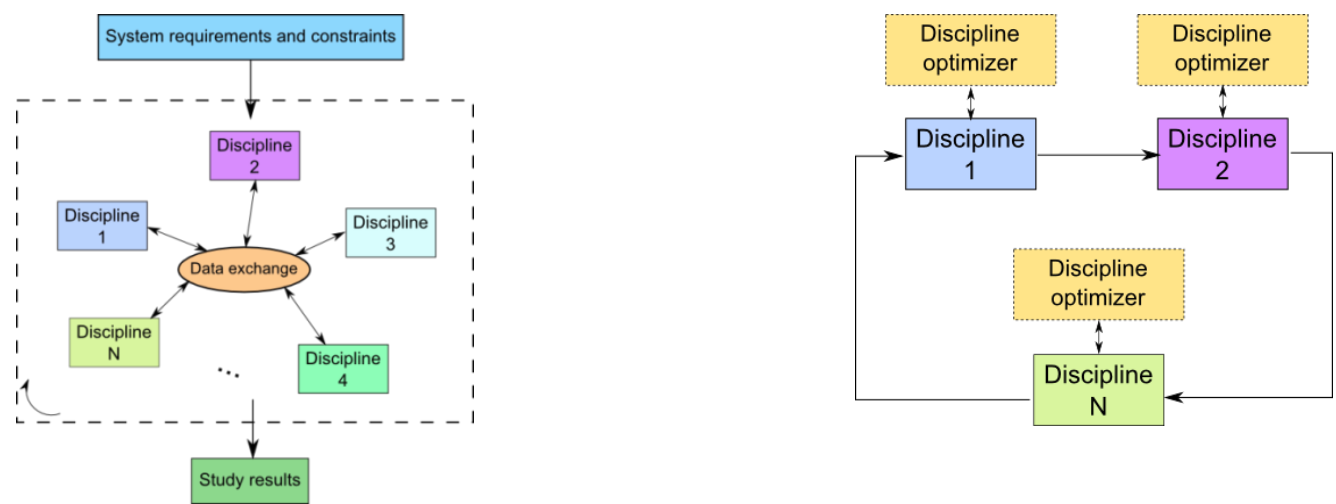

Figure 1: Concurrent engineering design process (left) and optimization (right) 
The existing concurrent engineering process (developed for instance at the Concurrent design Facility (CdF) of the European Space Agency, at the Centre d'Ingénierie Concourante of the Centre National d'Etudes Spatiales (CNES), in the space industry such as Airbus Safran Launcher) relies on an iterative process which involves engineering model interfaces (often through Microsoft Excel spreadsheets) handled by the field experts and a common data set used as inputs for the engineering models (Figure 1) (Xu et al., 2013). At each iteration of the concurrent engineering design process, the data set of each field is updated with the results from the discipline models and collaborative work sessions are organized. This approach offers advantages in terms of collaborative work and is a first approach in terms of interaction management between the disciplines. However, this classical concurrent engineering approach has several limitations:

- Use of important design margins in order to ensure multidisciplinary consistency between the disciplines,

- Difficulty to implement an optimization process at the system level. In classical concurrent engineering approach, the system design results from the concatenation of local disciplinary optimizations (Figure 1). However, due to possible antagonistic discipline objectives, a difficult search for compromise between these conflicting tasks needs to be performed.

- Difficulty to implement system level analyses (uncertainty propagation, reliability assessment, sensitivity analysis, optimization, etc.) in an automated process which could offer more information to the decision makers earlier in the design process.

In order to overcome the existing concurrent engineering limitations, a paradigm shift in terms of design process and analysis tools is necessary and Multidisciplinary Design Optimization (MDO) methodologies could be a key enabler to extend concurrent engineering capabilities. MDO is a collection of engineering methodologies to optimize systems modeled as a set of coupled disciplinary analyses. For instance, launch vehicles are customarily decomposed into interacting submodels for aerodynamics, trajectory, propulsion, mass and structure. The design of launch vehicle consists in determining its architecture to achieve a given mission and this launcher architecture is usually found through an optimization problem solving. Taking into account the different disciplines requires modelling and managing the interactions between them all along the optimization process.

Different topics related to MDO methodologies to facilitate aerospace vehicle design have been investigated:

a. Deterministic classical MDO formulations,

b. Deterministic dedicated MDO formulations relying on transversal decomposition of the design process,

c. MDO formulations under uncertainty in order to ensure the reliability and robustness with respect to uncertainty.

This paper is focused on several examples of recent use of MDO techniques at ONERA in order to illustrate the progress in terms of MDO methodologies. In the rest of the paper, these three topics will be explored relying on illustrative example on launch vehicle design problem. In section 2 , both classical and dedicated deterministic MDO methods for launch vehicle design are explored. In section 3, MDO methodologies in the presence of uncertainty are presented in order to ensure the reliability and robustness of the launch vehicles. In section 4, perspectives to use MDO methodologies for concurrent engineering sessions are discussed.

\section{DETERMINISTIC MDO METHODOLOGIES FOR LAUNCH VEHICLE DESIGN}

\section{a. Classical MDO formulations}

The design space exploration to design a launch vehicle requires formalizing the optimization problem to solve, meaning from the system and mission requirements, to identify the disciplines involved, the interactions and couplings between the disciplines, the design variables, the objective 
function, the constraints, etc. The aim is to build a design process that is compliant with certain requirements: robustness of the performance, computational cost of the process, dimension of the design space, or the constraints to be fulfilled. A major difference with the classical concurrent engineering approach is that in addition to share a common data set, the disciplinary models are chained offering possibility to account for interdisciplinary couplings and to ensure consistency between the discipline executions. Several organizations of the optimization process have been proposed, called MDO formulations (Figure 2). These formulations may be classified according to four categories (Balesdent et al., 2012a):

- Coupled formulations: easiest approaches, ensure interdisciplinary coupling consistency at each iteration of the optimization process (e.g. Multi Discipline Feasible (R. J. Balling et al., 1996)),

- Decoupled formulations: enable parallel discipline evaluations and ensure interdisciplinary coupling consistency at the convergence of the optimization problem (e.g. Individual Discipline Feasible (Balling et al., 1996)),

- Single-level formulations: the optimization process is driven by a single optimizer at the system-level (e.g. All At Once (Balling et al., 1996)),

- Multi-level formulations: the optimization process is driven by one optimizer at the systemlevel associated with several optimizers at the sub-system level (discipline level) in order to ease the system level optimizer convergence (e.g. Collaborative Optimization (Braun et al., 1996), Analytical Target Cascading (Allison et al., 2005)).

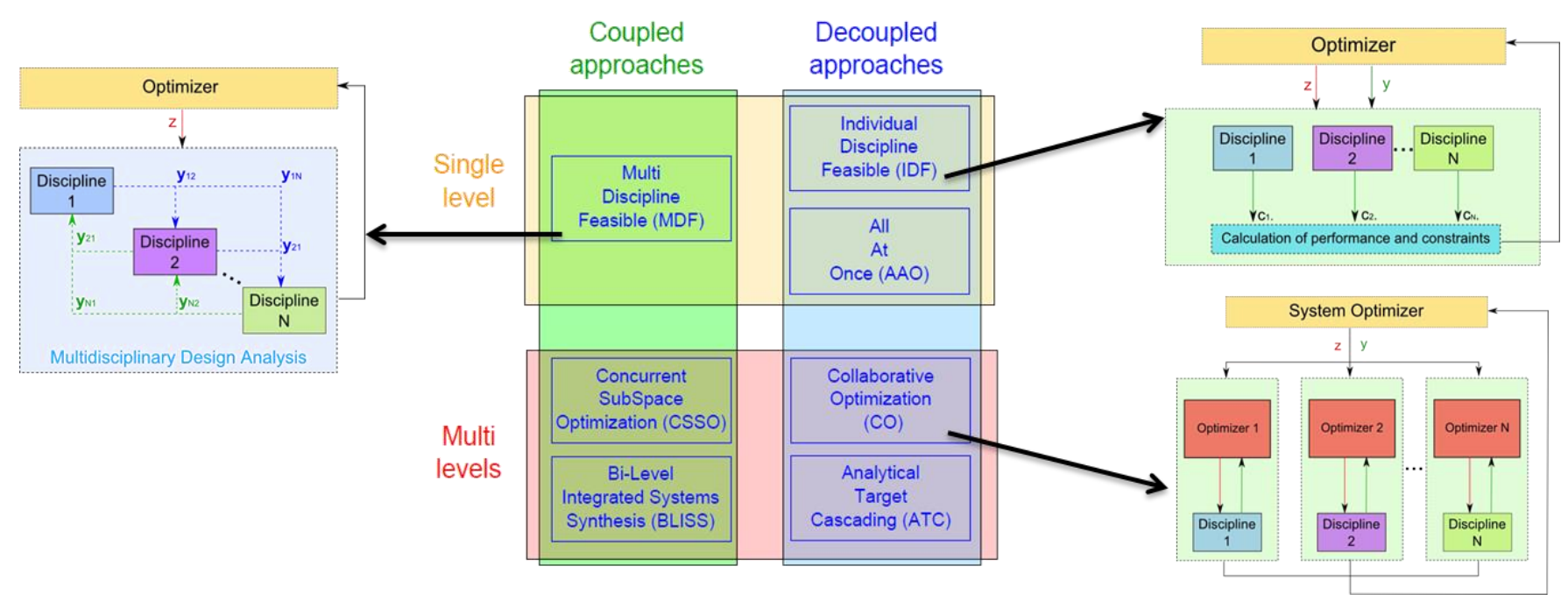

Figure 2: Classical general MDO formulations in the literature

These formulations have the advantage of being generic and adaptable to any complex system design. In order to illustrate how these global design issues could be taken into account to provide decision-makers a full understanding of the choices at stake, a system study was set up at ONERA, using internal facilities and design methods. This study explores the impact of reusability onto a global launcher design.

The starting point of this study is threefold:

- First, the baseline architecture of the current Ariane 6 design is considered as a starting point. The study logic is to try to derive a reusable launcher (with $1^{\text {st }}$ stage recovery) from this configuration, with minimal architecture and technology changes.

- Second, the $1^{\text {st }}$ stage recovery is assumed to be performed by a "space X-like" vertical landing on an infrastructure located in the ocean, and using only rocket propulsion during re-entry.

- Third, the main design assumption will be the use of a 80-ton-thrust LOX/CH4 engine, which has been stated as a promising solution.

These assumptions are not established to be the optimal ones, but provide a common reference to study several launcher variants (Figure 3). 

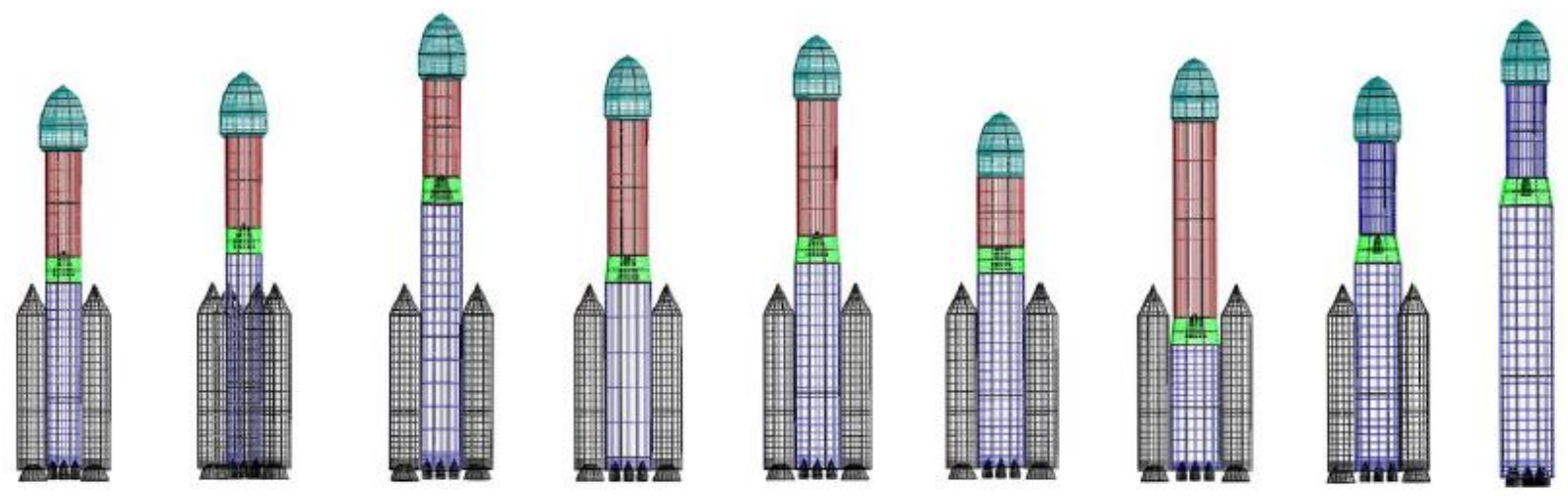

Figure 3: Resulting optimal configurations for 5-8t mission in GTO with a reusable first stage (blue: LOX/CH4, red: LOX/LH2, black: solid propulsion, named from left to right EA, EB, R1A, R1B, R1C, R2A, R2B, R3A, R3C)

From this starting point, the two stage launcher is fully redesigned to meet a delivery capability of 5 tons on a Geostationary Transfer Orbit $(250 \mathrm{~km} \times 36000 \mathrm{~km})$ with a minimal Gross Lift-Off Weight (GLOW). Several different configurations have been explored and optimized (Figure 3). A fastresponse sizing loop relying on a Multi Discipline Feasible (MDF) formulation was used to jointly optimize the following design variables:

- the launcher geometry (stage diameters, volume of propellants),

- the propulsion system (chamber pressure, nozzle expansion ratio, mixture ratio)

- the 3-phase trajectory (two-stage ascent with possible ballistic phases, controlled by pitch angle management, and descent, controlled by a pitch angle law and the duration and intensity of propulsion boosts; and reentry).

The disciplines involved are the propulsion, the mass budget and geometry, the aerodynamics and the trajectory. The discipline models are provided by field experts and interdisciplinary couplings between the disciplines have been identified in order to enable code chaining and to account for representative interdisciplinary interactions.

A MultiDisciplinary Analysis (MDA) based on iterative fixed point method is used to satisfy the interdisciplinary couplings at each iteration of the system level optimizer, ensuring the physical relevance of the designed launch vehicle. MDF is used to find the optimal configuration of the launcher, satisfying the design and interdisciplinary coupling constraints.
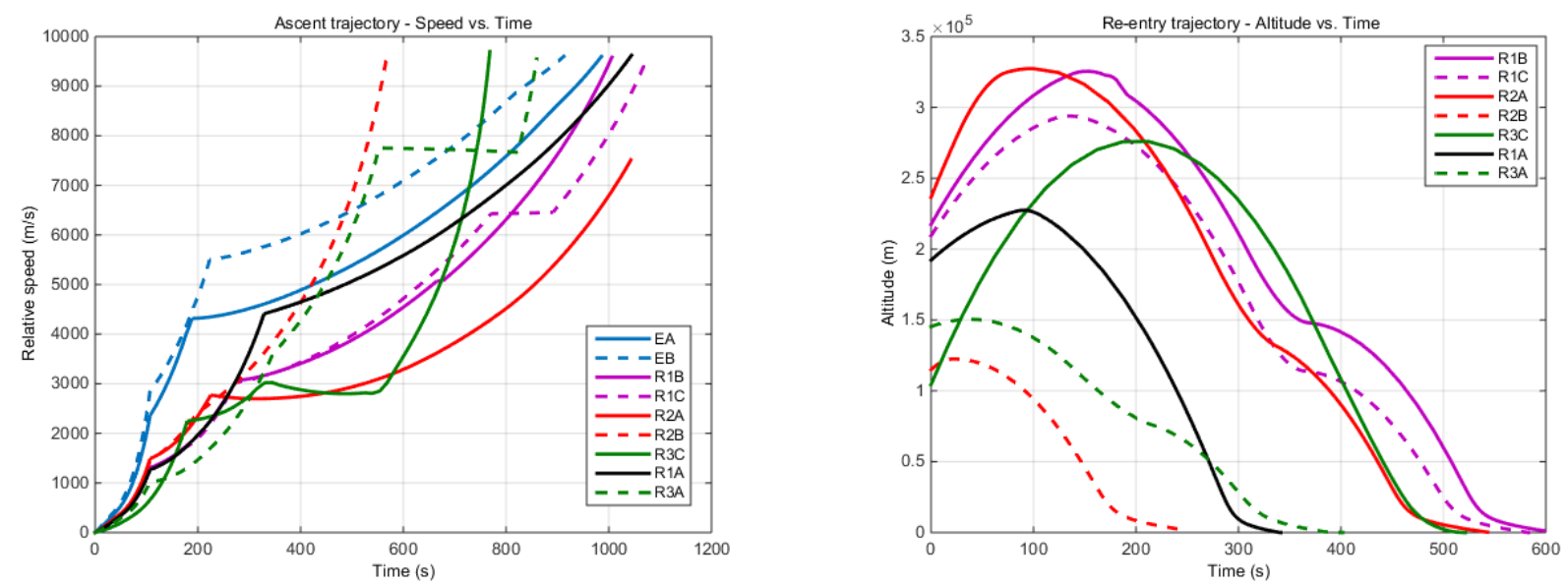

Figure 4: Ascent velocity and descent trajectory for different concepts 
After MDF runs, analyses with field experts and system engineers is performed in order to understand the design choices and to analyse the interdisciplinary interactions and their aftermaths on the design process. As a main outcome of this study, it appears that providing the $1^{\text {st }}$ stage with a recovery capability induces a full redesign of the launcher (i.e. propulsion choices, both from the propellant and the engine points of view, staging, trajectory). A trade-off is observed between:

- the staging Mach number (the higher, the easier the design of the upper stage, but the greater the re-entry difficulties),

- the propellant mass used for $1^{\text {st }}$ stage recovery (the lower, the better for global performance, but the higher the re-entry loads and thus the structural impacts),

- the design peak dynamic pressure during re-entry (the higher, the lower the fuel consumption, but the harder the loads on the re-used stage).

The order of magnitude of GLOW penalty to provide the launcher with reusability seems to be around $25 \%$, which is to be translated into cost increase for the same payload, and compared to the gain of reusability. Nevertheless, it seems that an easy derivation of a reusable version from current Ariane 6 concepts is not straightforward, and that maximum reuse of existing stages (boosters or upper stage) or even existing engines (Vinci or Vulcain) is doubtful or should be consolidated.

From the design point of view, due to the antagonistic discipline objectives (for instance ascent and descent trajectories), classical concurrent engineering approach does not offer the possibility to find global optimal solution. Moreover, designing the entire vehicle to provide new capability (here reusability of the $1^{\text {st }}$ stage) impacts all the disciplines through the couplings and the technological choices. Therefore, using a MDO approach enables to take into account the coupling effects between the different physics all along the design process and analysis which is a very important aspect for such complex system design. To quantify their performance and reliability tightly coupled multidisciplinary has been used in order to provide key decision arguments to the stakeholders In conclusion, MDO approach offers key advantages compared to concurrent engineering methods such as the possibility to explore large variety of configurations in parallel (nine concepts in this example) to assess the best architecture relatively to mission requirements.

\section{b. SWORD: Stage-Wise decomposition for Optimal Rocket Design}

The classical MDO formulations introduced in the previous section are flexible enough to be adapted to the design of any complex system such as launch vehicle. However, launch vehicle design present particularities, notably the importance of the trajectory discipline compared to the other disciplines. Exploiting these specificities in a dedicated MDO formulation might improve the launch vehicle design process. Specific formulations for launch vehicle design have been proposed such as the Stage-Wise decomposition for Optimal Rocket Design (SWORD) (Balesdent et al., 2012b). The classical MDO approaches as illustrated in the previous paragraph, decompose the design process according to the involved disciplines (propulsion, aerodynamics, sizing, trajectory, etc.).

In order to lay the trajectory optimization at the center of the MDO process, SWORD splits up the launch vehicle design problem according to the different flight phases and transforms the global MDO problem into the coordination of smaller ones. Each stage is optimized separately and the different stages are coordinated through the trajectory optimization, via the state vectors at the stage separations. In the SWORD formulations, the subsystems are not the disciplines but instead the different launch vehicle stage optimizations incorporating all the required disciplines to the stage design. Four different formulations have been proposed depending on the decomposition process and the interdisciplinary coupling constraint handling. One of the SWORD formulations consists of a hierarchical decomposition of the design process according to the launch vehicle stages (Figure 5). In this formulation, the objective function is assumed to be decomposed according to the different stages. In practice, the GLOW is often minimized in launch vehicle design process (Balesdent et al., 2012a) and it can be decomposed as the sum of the stage masses plus the fairing and payload masses. The different stage are coupled, a change in the upper stage mass would 
introduce a modification of the lower stage masses in order to ensure mission success. Also, the cost of launch vehicle follows the same decomposition (i.e. sum of the stage cost).

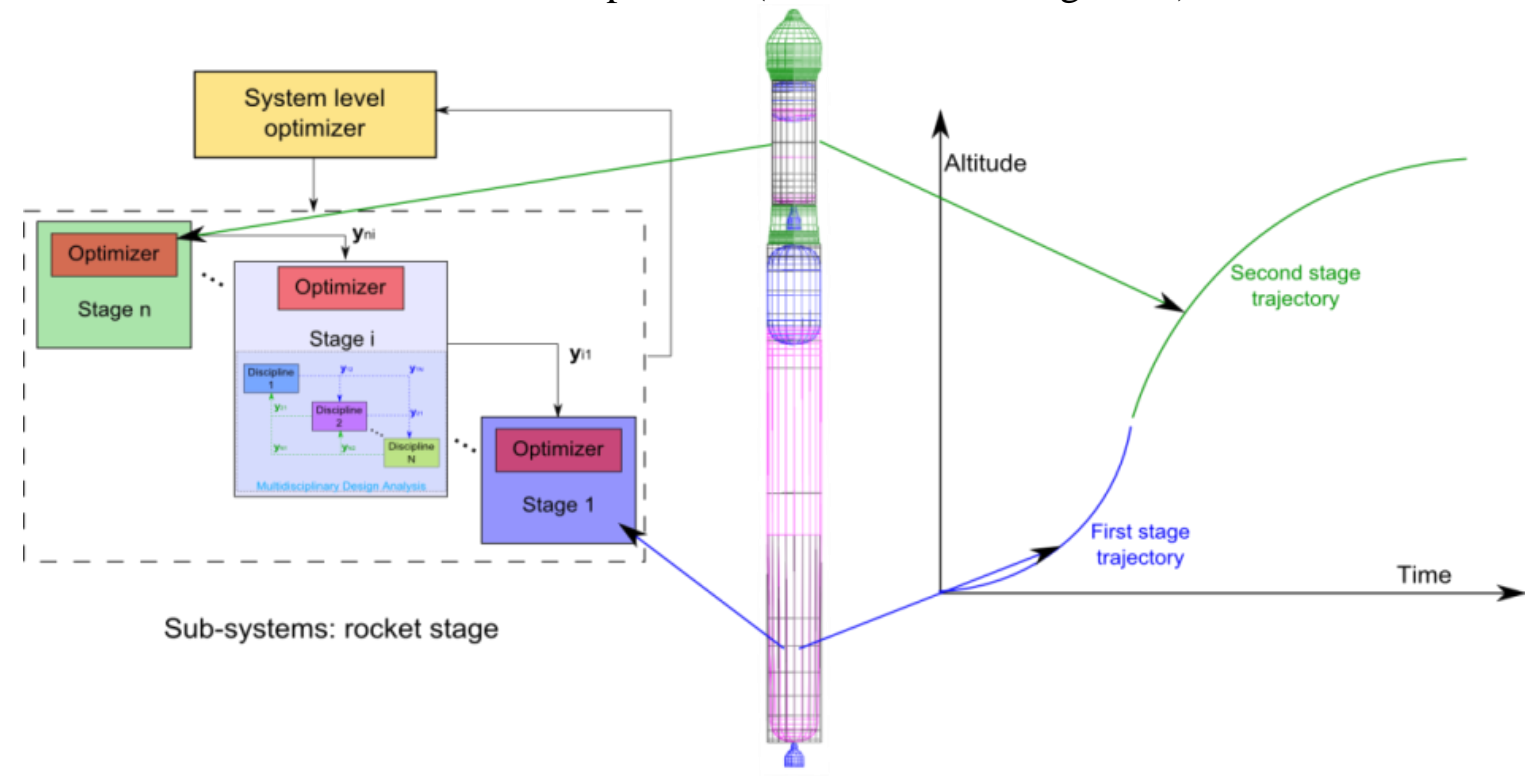

Figure 5: Example of SWORD formulation

In order to assess the efficiency of SWORD, a comparison with MDF formulation has been performed on a launch vehicle design problem using the same classical Genetic Algorithm in a very large search space. The problem to be solved is the optimization of a three-stage cryogenic launch vehicle including 40 design variables (trajectory command law, propellant masses, stage diameters, propulsion chamber pressures, etc.) (Balesdent et al., 2011). The objective function to be minimized is the GLOW. The payload mass is equal to 4 tons and the target orbit is a GTO. Three comparative criteria for the formulations have been selected:

- The best found design at the stopping time of the optimization algorithm;

- The time elapsed to find a first feasible design from random initialization;

- The improvement of the objective function during the optimization process.

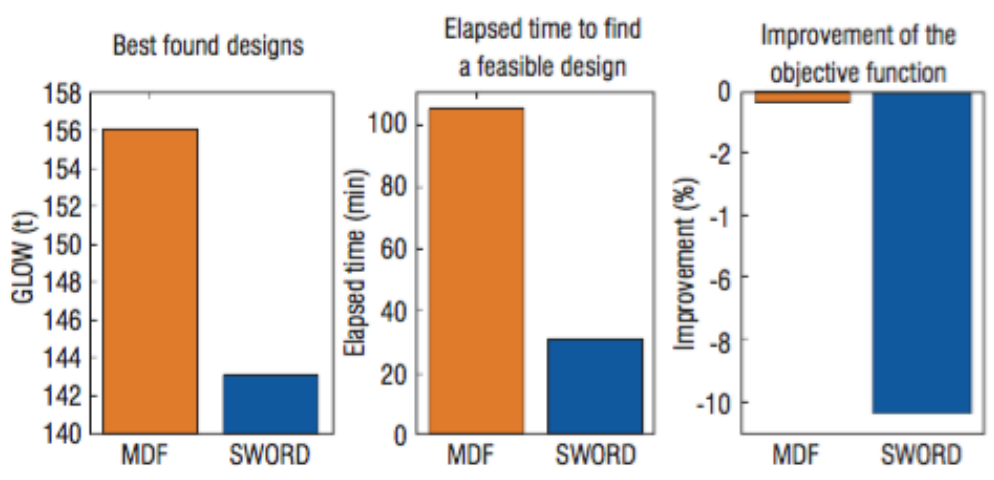

Figure 6: Comparison of SWORD and MDF (average over 10 optimizations)

SWORD clearly outperforms MDF in the case of global search optimization in a very large search space (Figure 6). SWORD allows to significantly improve the global search efficiency with respect to the MDF formulation by reducing in average by 4 the required computation time to find a first feasible design, finds a lighter design than MDF and allows to efficiently improve the current design all along the optimization process whereas MDF presents some difficulties to improve the objective function. SWORD is interesting as it ensures the consistency of the mass coupling during the optimization process and does not require equality constraints at the system level. 


\section{MDO METHODOLOGIES UNDER UNCERTAINTY FOR LAUNCH VEHICLE DESIGN}

The early design phases of LV are often characterized by the use of low fidelity analyses as well as by the lack of knowledge about the future system design and performance. Indeed, the low fidelity analyses are employed due to the non-possibility to build high fidelity models for each possible architecture to explore the entire design space. This global exploration results in repeated discipline evaluations which are impossible to perform at an affordable computational cost with high fidelity models. Moreover, to increase the performance of the launch vehicles and to decrease their costs, space agencies and industries introduce new technologies (new propellant mixture such as liquid oxygen and methane, reusable rocket engines) and new architectures (reusable first stage for launch vehicles) which present a high level of uncertainty in the early design phases. Incorporating uncertainties in MDO methodologies for aerospace vehicle design has thus become a necessity to offer improvements in terms of reduction of design cycle time, costs and risks, robustness of launch vehicle design to with respect to uncertainty along the development phase, and increasing the system performance while meeting the reliability requirements.

If uncertainties are not taken into account at the early design phases, the detailed design phase might reveal that the optimal design previously found violates specific requirements and constraints. In this case, either the designers go back to the previous design phase to find a set of design alternatives, or they perform design modifications at the detailed design phase that could result in loss of performance. Both options would result in a loss of time and money due to the rerun of complex simulations. Moreover, uncertainties are often treated with safety margins during the design process of launch vehicles which may result in over conservative designs therefore an adequate handling of uncertainty is essential (Brevault et al. 2015). Uncertainty-based Multidisciplinary Design Optimization (UMDO) aims at solving MDO problems under uncertainty.

Incorporating uncertainty in MDO methodologies raises a number of challenges which need to be addressed. Being able, in the early design phases, to design a multidisciplinary system taking into account the interactions between the disciplines and to handle the inherent uncertainties is often computationally prohibitive. In order to satisfy the designer requirements, it is necessary to find the system architecture which is optimal in terms of system performance while ensuring the robustness and reliability of the optimal system with respect to uncertainty. One of the key challenges is the handling of interdisciplinary couplings in the presence of uncertainty. Most of the existing UMDO formulations are based on an adaptation of the single-level MDF formulation in the presence of uncertainty (Brevault et al., 2015). These methods are very computationally expensive because they combine the computational cost of the optimisation problem solving, the interdisciplinary coupling by using an MDA (i.e. loop between the disciplines) and the uncertainty propagation often performed by Monte Carlo simulations. Alternatives exist (e.g. SORA (Du et al., 2004)) but introduce simplifications that can lead to wrong design.

In order to tackle the computational cost of coupled MDO formulations and preserve the validity of the found design, two new UMDO formulations (Brevault et al. 2015, Brevault et al. 2016) with interdisciplinary coupling satisfaction for all the realizations of the uncertain variables have been elaborated. With the aim of ensuring multidisciplinary feasibility, a new technique has been proposed based on a parametric surrogate model (Polynomial Chaos Expansion) of the input coupling variables and a new interdisciplinary coupling constraint to guarantee the validity of the of the interdisciplinary coupling satisfaction when impacted by uncertainties. This technique enables the system-level optimizer to control the parameters defining the surrogate model of the input coupling variables in addition to the design variables. Therefore, it enables to decouple the disciplines while ensuring at the UMDO problem convergence that the functional relations between the disciplines are the same as if a coupled approach using MDA had been used. The two proposed formulations rely on this technique to handle interdisciplinary couplings. The first formulation is a 
single-level approach inspired from Individual Discipline Feasible (IDF) and adapted to the presence of uncertainty. This approach, called Individual Discipline Feasible - Polynomial Chaos Expansion (IDF-PCE) (Brevault et al. 2015) (Figure 7), allows to ensure multidisciplinary feasibility for the optimal solution while reorganizing the design process through a decomposition strategy.
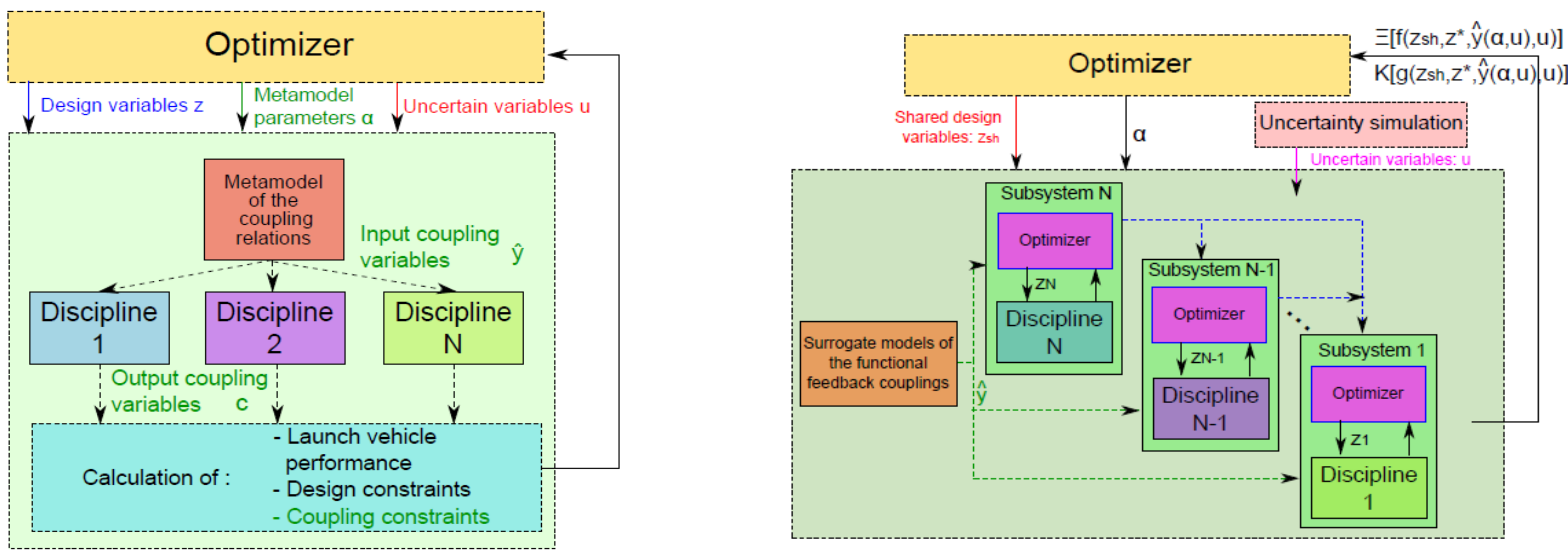

Figure 7: IDF-PCE (left) and MHOU (right) formulations

The second formulation is a multi-level approach inspired from SWORD (Balesdent et al., 2012b), which has been modified to take into account uncertainty and to maintain the equivalence with coupled approaches in terms of multidisciplinary feasibility. This formulation (Figure 7), named Multi-level Hierarchical Optimization under Uncertainty (MHOU) (Brevault et al. 2015), introduces multi-level optimization of the disciplines and is particularly adapted for launch vehicle design.

These formulations have been applied on a two stage launch vehicle design test problem and compared to the MDF under uncertainty formulation. Four coupled disciplines are involved in this problem: the aerodynamics, the mass budget, the propulsion and the trajectory (Figure 8). This test case consists in minimizing the expected value of the GLOW under the constraint of injecting a $5 \mathrm{t}$ payload into a GTO orbit at the perigee of $250 \mathrm{~km}$. The design problem has 27 design variables (stage diameter, propellant mass, thrust, mixture ratio, derating factor, trajectory control law) and three uncertain variables are taken into account $\left(1^{\text {st }}\right.$ stage specific impulse uncertainty, $2^{\text {nd }}$ stage thrust uncertainty and $2^{\text {nd }}$ stage dry mass uncertainty). The probability of failure to reach the target orbit is estimated by Subset Simulation (Au et al., 2001) using Support Vector Machine (Dubourg et al., 2013) of the limit state defining the failure. A patternsearch algorithm (Audet et al., 2002) is used for the system level optimizer. 


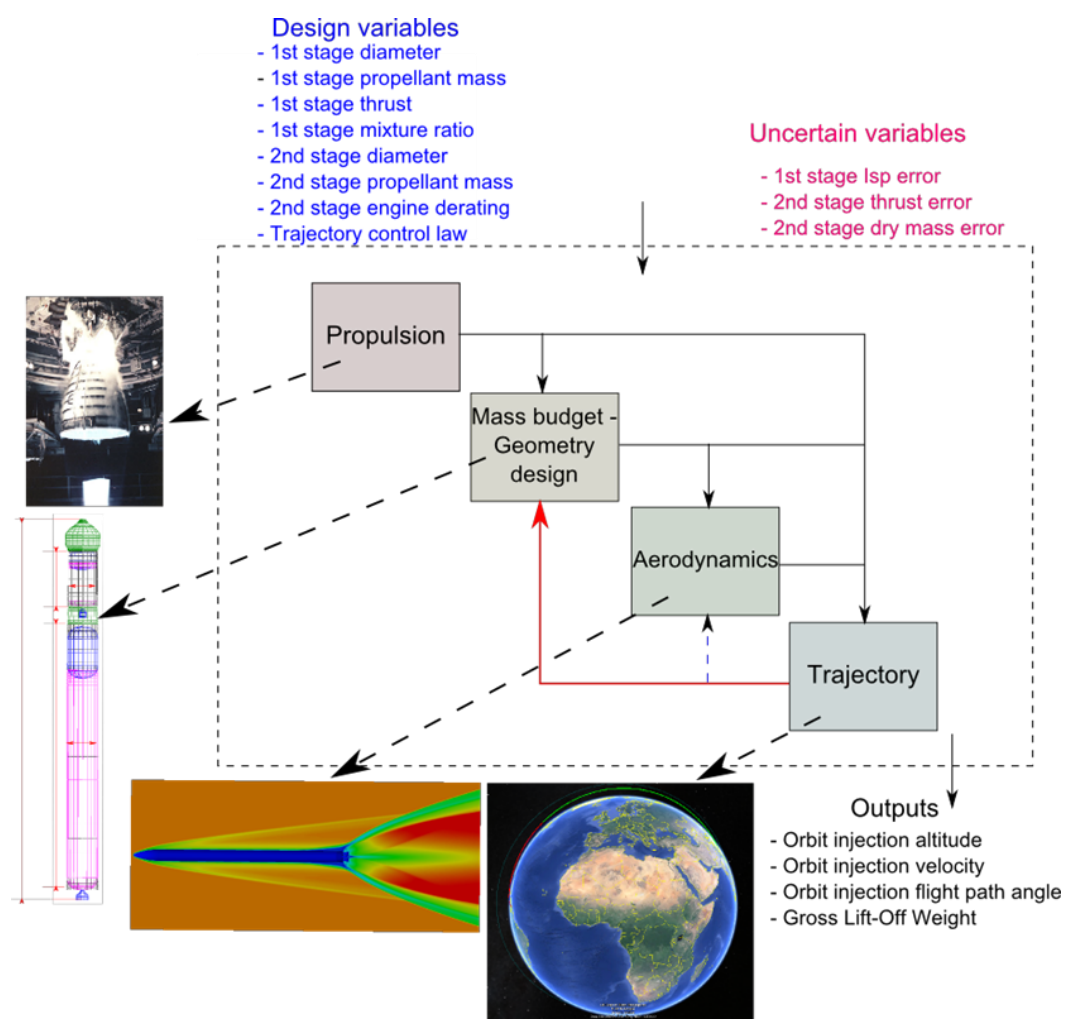

Figure 8: MultiDisciplinary Analysis for the launch vehicle design problem under uncertainty
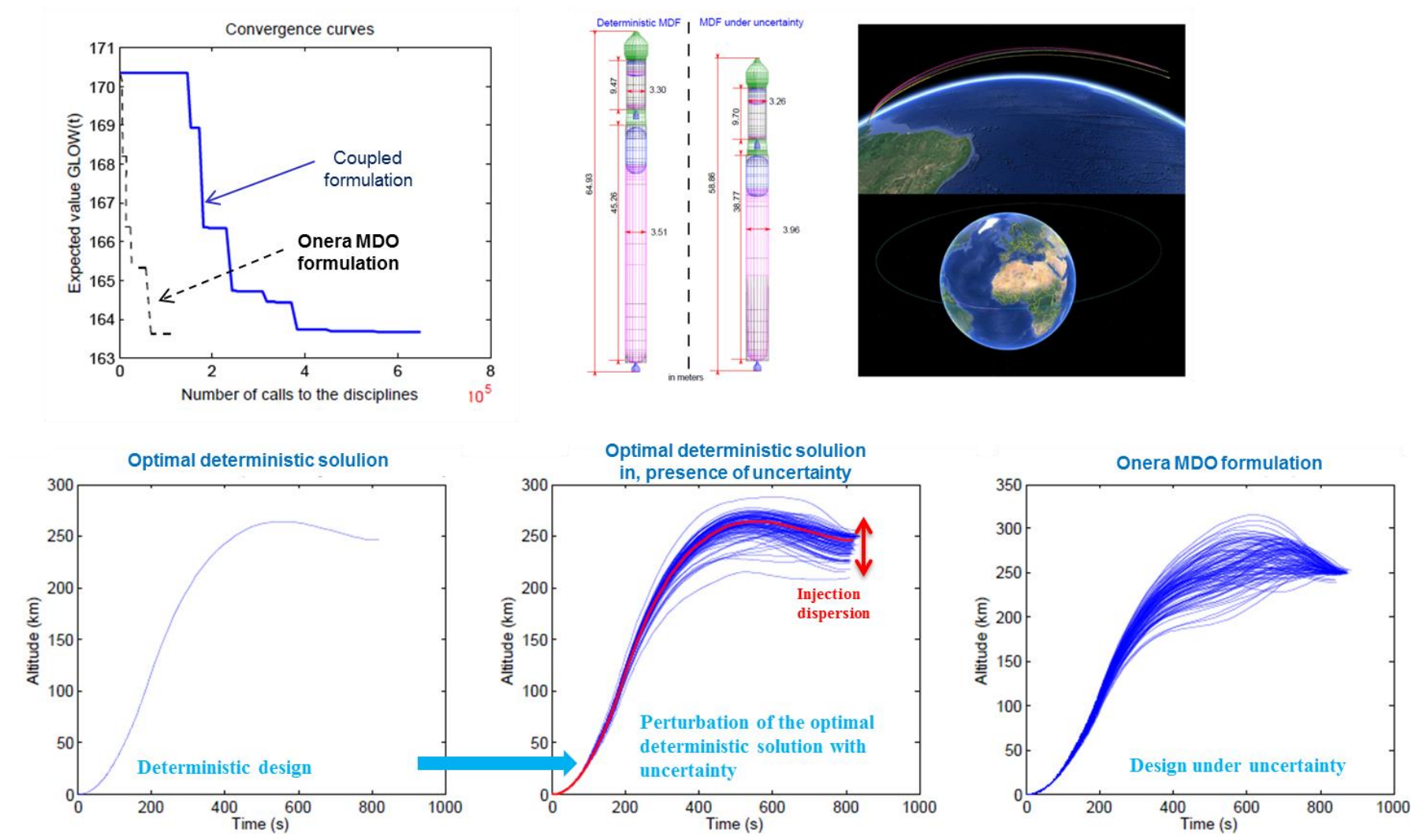

Figure 9: MDO under uncertainty on a launch vehicle design test case

As presented in Figure 9 (upper left), the ONERA MDO formulation (the decoupled single-level IDF-PCE) under uncertainty is more efficient (by a factor of 11) in terms of number of calls to the different disciplines than the coupled approach MDF under uncertainty. The importance of taking into account the presence of uncertainty is highlighted in the bottom of Figure 9. Indeed, the same 
problem has been solved with a deterministic approach (considering all the uncertainties frozen at their expected values, bottom left of Figure 9). An optimal launch vehicle has been found with a corresponding trajectory. Then, the optimal deterministic vehicle has been perturbed by the presence of uncertainties and has resulted in an important injection dispersion of the payload into orbit (bottom middle Figure 9). As illustrated on the bottom right of Figure 9, taking into account the presence of uncertainty directly in the design process enables to design a vehicle that is robust to to uncertainty allowing to ensure the required injection precision for the payload. Using a MDO approach enables to perform in a simple manner uncertainty propagation and optimization while taking into account the potential cascading effects on the different disciplines through the interdisciplinary couplings.

\section{CONCLUSIONS}

In order to develop innovative aerospace vehicle concepts it is necessary to achieve increasingly complex system integration studies and one faces the challenge of developing efficient, robust and adapted design methodologies. Comprehensive study of process decomposition, uncertainty quantification, high-fidelity tool integration and formulation of the optimization strategy are mandatory, but this theory must always be tested and validated on 'real-life' design cases.

To enhance collaborative efficiency between system engineers, disciplinary experts, CAD designers and decision makers concurrent engineering should rely on dedicated methodologies for system design studies. As illustrated in Sections 2 and 3 Multidisciplinary Design Optimization tools could enhance concurrent engineering approach by enabling fully integrated multidisciplinary analysis to ensure appropriate interdisciplinary consistency while allowing to assess system tradeoff between antagonist discipline objectives. Concurrent engineering sessions could be improved by setting a multidisciplinary collaborative framework enabling to quickly assess, compare and submit to experts and decision makers the different concepts under evaluation. To do so, a proper description of models, interdisciplinary couplings, data exchange process, objective functions and constraint functions is required in order to appropriately formulate the MDO problem. Moreover, capitalization of disciplinary models and libraries of methodologies are required to develop such a framework. Using MDO methodologies between concurrent engineering sessions would enable to analyze the system performance with the disciplinary experts focusing on the interdisciplinary couplings improving the understanding of the impact of each discipline on the system performance.

From a design capability point of view, the combined use of an integrated MDO approach (for instance for launch vehicle design, the whole interactions between disciplines being modelled, and a joint geometry/trajectory optimization being performed) and a collaborative environment (allowing exchanges between experts and design space visualization and exploration) enables to explore quickly several design options and provide sensitivities. As illustrated in this paper, dedicated MDO formulations for launch vehicle design are more efficient in terms of quality of the found solution and computational cost than classical integrated approach. Moreover, the presented results outlined the importance of taking into account uncertainties in the early design phases in order to incorporate the lack of knowledge and to design robust systems.

Further improvements in MDO methodologies are required to be applied on difficult industrial problems. For instance, MDO for high dimensional problems (e.g. high dimensional couplings such as aerodynamic and structure meshes) is still a major challenge. Another important issue is the appropriate handling of mixed continuous, discrete and categorical variables in MDO problems in order to make technological choices along with system performance optimization. Development of new methodologies is required to tackle these types of problems in order to be applied on complex industrial problems.

\section{ACKNOLEDGMENT}

This work has been partially supported by CNES. This support is gratefully acknowledged. 


\section{REFERENCES}

J. Allison, M. Kokkolaras, M. Zawislak, and P.Y. Papalambros, (2005), On the Use of Analytical Target cascading and Collaborative Optimization for Complex System Design, International Society for Structural and Multidisciplinary Optimization, AIAA and the Brasilian Soc. Of Mechanical Sciences and Engineering, Rio de Janeiro, 2005, pp. 3091-3100.

S. Au, J. Beck (2001) Estimation of small failure probabilities in high dimensions by subset simulation. Probabilistic Eng Mech 16(4):263-277. doi:10.1016/S0266-8920(01)00019-4

C. Audet, and J.E. Dennis, Jr.,Analysis of Generalized Pattern Searches, SIAM Journal on Optimization, Vol. 13, No. 3, 2002, pp. 889-903. doi:10.1137/S1052623400378742

M. Balesdent (2011), Multidisciplinary Design Optimization of launch vehicles. PhD Thesis, Ecole Centrale de Nantes.

M. Balesdent, N. Bérend, Ph. Dépincé and A. Chriette (2012a) A Survey of Mutidisciplinary Design Optimization methods in Launch Vehicle Design, Structural and Multidisciplinary Optimization, Springer. Vol.45(5):619-642 DOI : 10.1007/s00158-011-0701-4.

M. Balesdent, N. Bérend and Ph. Dépincé (2012b) Stagewise Multidisciplinary Design Optimization Formulation for Optimal Design of Expendable Launch Vehicles, Journal of Spacecraft and Rockets, AIAA. Vol. 49(4):720-730 DOI : 10.2541/1.52507

M. Balesdent, G. Ordonneau, S. Defoort, L. Brevault, T. Donath (2015) Launcher reusability: preliminary study on propulsion and system impacts - An illustration of ONERA's knowledge and tools, ONERA internal report (RT 1/24949 DCPS)

R. J. Balling, and Sobieszczanski-Sobieski (1996), Optimization of Coupled Systems - A Critical overview of Approaches, AIAA Journal, Vol. 34, No. 1, 1996, pp. 6-17. doi:10.2514/3.13015

R. D. Braun, A.A. Moore, I.M. Kroo, (1996), Use of the Collaborative Optimization Architecture for Launch Vehicle Design, 6thAIAA/USAF/NASA/ISSMO Symposium on Multidisciplinary Analysis and Optimization, Part I, AIAA Paper 1996-4018, 1996, pp. 306-318.

L. Brevault, M. Balesdent, N. Bérend and R. Le Riche (2016) Decoupled MDO formulation for interdisciplinary coupling satisfaction under uncertainty, AIAA Journal, Vol. 54(1) : 186-205, DOI : $10.2514 / 1 . J 054121$

L. Brevault, M. Balesdent, N. Bérend and R. Le Riche (2015) Multi-level Hierarchical MDO formulation with functional coupling satisfaction under uncertainty, application to sounding rocket design. $11^{\text {th }}$ ISSMO World Congress on Structural and Multidisciplinary Optimization, 7-12 June, 2015, Sydney, Australia

L. Brevault, (2015) Multidisciplinary Design Optimization under uncertainty, application to launch vehicle design. PhD. Thesis. Ecole Nationale Supérieur des Mines de Saint-Etienne.

A. Chaudhuri, K. Willcox. (2016). Multifidelity Uncertainty Propagation in Coupled Multidisciplinary Systems. 18th AIAA Non-Deterministic Approaches Conference.

G. Dhondt, CalculiX CrunchiX User's Manual, version 2.9, November 19, 2015

X. Du, W. Chen. (2004) Sequential optimization and reliability assessment method for efficient probabilistic design. Journal of Mechanical Design 126(2):225-233

V. Dubourg, B. Sudret, and F. Deheeger, Metamodel-Based Importance Sampling for Structural Reliability Analysis, Probabilistic Engineering Mechanics, Vol. 33, July 2013, pp. 47-57. doi:10.1016/j.probengmech.2013.02.002

C. Heath, and J. Gray (2012), OpenMDAO: Framework for flexible multidisciplinary design, analysis and optimization methods, Proceedings of the 53rd AIAA Structures, Structural Dynamics and Materials Conference, Honolulu, HI 
L. Jaeger, C. Gogu, S. Segonds, and C. Bes (2013) Aircraft Multidisciplinary Design Optimization Under Both Model and Design Variables Uncertainty, Journal of Aircraft, Vol. 50, No. 2, pp. 528-538.

P. Jiang, Q. Zhou, X. Shao, R. Long, H. Zhou. (2016) A modified BLISCO method and its combination with variable fidelity metamodel for engineering design. Engineering Computations 33:5, 1353-1377

M. Moore, OpenVSP User Manual, Version 1.7.92, Langley Research Center, NASA, 2015

D. Myers (1982), Matrix formulation of co-kriging, Journal of the International Association for Mathematical Geology, 14(3), pp. 249-257

D. Xu, C. Bil, and al. (2013) Overview on the development of concurrent design facility \}, 20th ISPE International Conference on Concurrent Engineering: Proceedings, pp. 551

Q. Zhou, X. Shao, P. Jiang, Z. Gao, H. Zhou, L. Shu. (2016) An active learning variable-fidelity metamodelling approach based on ensemble of metamodels and objective-oriented sequential sampling. Journal of Engineering Design 27:4-6, 205-231 